\title{
A NOTE ON ASYMPTOTIC BEHAVIOR OF SOLUTIONS TO A HETEROGENEOUS NONLINEAR REACTION-DIFFUSION SYSTEM
}

\author{
SINING ZHENG
}

\begin{abstract}
A result on asymptotic behavior of solutions to a heterogeneous nonlinear reaction-diffusion system with homogeneous Neumann boundary condition is obtained, which improves the results in [5].
\end{abstract}

1. Introduction. The large time behavior of solutions to systems of nonlinear reaction-diffusion equations, namely, the decay to the spatially homogeneous solutions, was studied by E. Conway, D. Hoff, and J. Smoller [2]. The corresponding heterogeneous case was considered by Y. Su. [5]. This paper improves the main result of $[\mathbf{5}]$.

We consider the heterogeneous system

$$
u_{t}=\left(1 / \varepsilon^{2}\right) D \Delta u+f(x, t, u), \quad(x, t) \in \Omega \times \mathbf{R}^{+},
$$

with initial condition

$$
u(x, 0)=u_{0}(x), \quad x \in \bar{\Omega},
$$

and homogeneous Neumann boundary condition

$$
\frac{\partial u}{\partial n}(x, t)=0, \quad(x, t) \in \partial \Omega \times \mathbf{R}^{+},
$$

where $u \in \mathbf{R}^{n}, \Omega \subset \mathbf{R}^{m}$ is a bounded domain, $\partial \Omega$ is the boundary of $\Omega, \partial / \partial n$ represents the outward normal derivative on $\partial \Omega, D$ is an $n \times n$ matrix, $f: \Omega \times \mathbf{R}^{+} \times$ $\mathbf{R}^{n} \rightarrow \mathbf{R}^{n}$ is a nonlinear vector function, and $\varepsilon>0$ is a small parameter concerning the diffusion strength.

We will approximate the solutions of (1.1)-(1.3) by corresponding spatially homogeneous solutions and give the estimate of the "error".

2. Main result. We need the following assumptions:

(A-1) System (1.1) admits a bounded invariant region $[\mathbf{1}] \Sigma \subset \mathbf{R}^{n}$.

(A-2) The diffusion matrix $D$ is positive definite, i.e.,

$$
\langle D v, v\rangle \geq d|v|^{2} \quad \forall v \in \mathbf{R}^{n}
$$

where $d>0$ is the smallest (positive) eigenvalue of $D$.

(A-3) $f(x, t, u) \in C^{1,0,1}\left(\Omega \times \mathbf{R}^{+} \times \mathbf{R}^{n}\right)$, i.e., $f$ has bounded first order derivatives with respect $x \in \Omega$ and $u \in \Sigma$.

Received by the editors May 15, 1985 and, in revised form, August 12, 1985.

1980 Mathematics Subject Classification. Primary 35K60; Secondary 35B40.

(C)1986 American Mathematical Society $0002-9939 / 86 \$ 1.00+\$ .25$ per page 
Denote

$$
\begin{aligned}
M & =\max \left\{\left|d f_{u}\right|: u \in \Sigma, x \in \bar{\Omega}, t \in \mathbf{R}^{+}\right\}, \\
N(t) & =\max \left\{\left|d f_{x}\right|: u \in \Sigma, x \in \bar{\Omega}\right\}, \\
\bar{u}(t) & =\frac{1}{|\Omega|} \int_{\Omega} u(x, t) d x, \\
\bar{f}(t, u) & =\frac{1}{|\Omega|} \int_{\Omega} f(x, t, u) d x, \\
\sigma & =d \lambda-M \varepsilon^{2},
\end{aligned}
$$

where $\lambda>0$ is the principal eigenvalue of $-\Delta$ on $\Omega$ with homogeneous Neumann boundary condition, $|\Omega|$ is the measure of $\Omega$.

Our main result is

THEOREM. Assume (A-1)-(A-3) hold, $u_{0}(x) \in \Sigma$ and $\sigma>0$. Then the solution $u(x, t)$ of (1.1)-(1.3) satisfies

$$
\begin{aligned}
\|u(x, t)-\bar{u}(t)\|_{L^{2}(\Omega)} \leq & \lambda^{-1 / 2}\left\|\nabla u_{0}\right\|_{L^{2}(\Omega)} e^{-\sigma t / 2 \varepsilon^{2}} \\
& +\lambda^{-1 / 2} N(t)\left(\varepsilon^{2} / \sigma\right)|\Omega|^{1 / 2}, \quad t \in \mathbf{R}^{+},
\end{aligned}
$$

and $\bar{u}(t)$ satisfies

$$
\left\{\begin{array}{l}
\frac{d \bar{u}(t)}{d t}=\bar{f}(t, \bar{u})+p(t), \quad t \in \mathbf{R}^{+} \\
\bar{u}(0)=\frac{1}{|\Omega|} \int_{\Omega} u_{0}(x) d x
\end{array}\right.
$$

where

$$
|p(t)| \leq M \lambda^{-1 / 2}|\Omega|^{-1 / 2}|| \nabla u_{0} \|_{L^{2}(\Omega)} e^{-\sigma t / 2 \varepsilon^{2}}+M N(t) \lambda^{-1 / 2} \sigma^{-1} \varepsilon^{2}, \quad t \in \mathbf{R}^{+} .
$$

PROOF. Here we will use the following facts, the proof of which can be found in Appendix A of [2]:

For $w \in W_{2}^{2}(\Omega), \partial w /\left.\partial n\right|_{\partial \Omega}=0$, we have

$$
\begin{aligned}
\|\Delta w\|_{L^{2}(\Omega)}^{2} & \geq \lambda\|\nabla w\|_{L^{2}(\Omega)}^{2}, \\
\|\nabla w\|_{L^{2}(\Omega)}^{2} & \geq \lambda\|w-\bar{w}\|_{L^{2}(\Omega)}^{2} .
\end{aligned}
$$

Define

Then

$$
\varphi(t)=\frac{1}{2}\|\nabla u\|_{L^{2}(\Omega)}^{2} .
$$

$$
\begin{aligned}
\varphi^{\prime}(t) & =\int_{\Omega}\left\langle\nabla u, \nabla u_{t}\right\rangle d x=\int_{\Omega}\left\langle\nabla u, \nabla\left(\frac{1}{\varepsilon^{2}} D \Delta u+f\right)\right\rangle d x \\
& =-\int_{\Omega}\left\langle\Delta u, \frac{1}{\varepsilon^{2}} D \Delta u\right\rangle d x+\int_{\Omega}\left\langle\nabla u, d f_{u} \cdot \nabla u+d f_{x}\right\rangle d x \\
& \leq-\frac{d \lambda}{\varepsilon^{2}} \int_{\Omega}|\nabla u|^{2} d x+M \int_{\Omega}|\nabla u|^{2} d x+\delta \int_{\Omega}|\nabla u|^{2} d x+\frac{N^{2}}{4 \delta}|\Omega| \\
& =2\left(M-\frac{d \lambda}{\varepsilon^{2}}+\delta\right) \varphi(t)+\frac{N^{2}}{4 \delta}|\Omega| \\
& =2\left(-\frac{\sigma}{\varepsilon^{2}}+\delta\right) \varphi(t)+\frac{N^{2}}{4 \delta}|\Omega| .
\end{aligned}
$$


Put

Then we have

$$
\psi(t)=\varphi(t)+\left[2\left(-\frac{\sigma}{\varepsilon^{2}}+\delta\right)\right]^{-1} \frac{N^{2}}{4 \delta}|\Omega|
$$

and hence

$$
\psi^{\prime}(t) \leq 2\left(-\sigma / \varepsilon^{2}+\delta\right) \psi(t)
$$

$$
\psi(t) \leq \psi(0) e^{2\left(-\sigma / \varepsilon^{2}+\delta\right) t}
$$

Take $\delta=\sigma / 2 \varepsilon^{2}$, then $2\left(-\sigma / \varepsilon^{2}+\delta\right)=-\sigma / \varepsilon^{2}<0$. So

$$
\begin{aligned}
\varphi(t) & \leq\left(\varphi(0)-\frac{N^{2} \varepsilon^{4}}{2 \sigma^{2}}|\Omega|\right) e^{-\sigma t / \varepsilon^{2}}+\frac{N^{2} \varepsilon^{4}}{2 \sigma^{2}}|\Omega| \\
& \leq \varphi(0) e^{-\sigma t / \varepsilon^{2}}+\frac{N^{2} \varepsilon^{4}}{2 \sigma^{2}}|\Omega|,
\end{aligned}
$$

i.e.,

$$
\|\nabla u\|_{L^{2}(\Omega)}^{2} \leq\left\|\nabla u_{0}\right\|_{L^{2}(\Omega)}^{2} e^{-\sigma t / \varepsilon^{2}}+\frac{N^{2} \varepsilon^{4}}{\sigma^{2}}|\Omega|, \quad t \in \mathbf{R}^{+} .
$$

By using (2.5) we get

$$
\|u(x, t)-\bar{u}(t)\|_{L^{2}(\Omega)}^{2} \leq \lambda^{-1}\left\|\nabla u_{0}\right\|_{L^{2}(\Omega)}^{2} e^{-\sigma t / \varepsilon^{2}}+\frac{N^{2} \varepsilon^{4}}{\sigma^{2}} \lambda^{-1}|\Omega|, \quad t \in \mathbf{R}^{+},
$$

and hence obtain (2.1).

To prove (2.2) and (2.3), we have

$$
\begin{aligned}
\frac{d \bar{u}(t)}{d t} & =\frac{1}{|\Omega|} \int_{\Omega} u_{t}(x, t) d x=\frac{1}{|\Omega|} \int_{\Omega}\left(\frac{1}{\varepsilon^{2}} D \Delta u+f\right) d x \\
& =\frac{1}{|\Omega|} \int_{\Omega} f(x, t, u) d x \\
& =\frac{1}{|\Omega|} \int_{\Omega} f(x, t, \bar{u}) d x+\frac{1}{|\Omega|} \int_{\Omega}(f(x, t, u)-f(x, t, \bar{u})) d x \\
& \equiv \bar{f}(t, \bar{u})+p(t) . \\
|p(t)| & \leq \frac{1}{|\Omega|} \int_{\Omega}|f(x, t, u)-f(x, t, \bar{u})| d x \\
& \leq \frac{M}{|\Omega|}|\Omega|^{1 / 2}\left(\int_{\Omega}|u(x, t)-\bar{u}(t)|^{2} d x\right)^{1 / 2} \\
& =M|\Omega|^{-1 / 2}\|u(x, t)-\bar{u}(t)\|_{L^{2}(\Omega)} \\
& \leq M \lambda^{-1 / 2}|\Omega|^{-1 / 2}|| \nabla u_{0} \|_{L^{2}(\Omega)} e^{-\sigma t / 2 \varepsilon^{2}}+M N(t) \lambda^{-1 / 2} \sigma^{-1} \varepsilon^{2}, \quad t \in \mathbf{R}^{+} .
\end{aligned}
$$

This proves (2.2) and (2.3),

The proof of the theorem is completed.

COROLLARY 1. If $\lim _{t \rightarrow \infty} N(t)=0$, then

$$
\begin{aligned}
& \lim _{t \rightarrow \infty}\|u(x, t)-\bar{u}(t)\|_{L^{2}(\Omega)}=0, \\
& \lim _{t \rightarrow \infty}|p(t)|=0 .
\end{aligned}
$$

In particular, if $D$ is a diagonal matrix, then (2.6) can be strengthened to

$$
\lim _{t \rightarrow \infty}\|u(x, t)-\bar{u}(t)\|_{L^{\infty}(\Omega)}=0 .
$$


PROOF. (2.6) and (2.7) result from (2.1) and (2.3), respectively. The proof of (2.8) can be done as the same as that in Appendix B of [2].

COROLlaRY 2. If $f=f(t, u)$ instead of $f(x, t, u)$ in (1.1), then the asymptotic behavior of the solution $u(x, t)$ of (1.1)-(1.3) is the same as that in the case $f=$ $f(u)$, i.e., $u(x, t)$ decays to $\bar{u}(t)$ exponentially (see Theorem 3.1 of [2]).

PROOF. This is the straightforward result of our above theorem and Theorem 3.1 of $[2]$.

3. Remark. Our theorem improves the result of Theorem 1 of [5]. First, condition $2 d \lambda-(2 M+1) \varepsilon^{2}<0$ in [5] is weakened to $d \lambda-M \varepsilon^{2}<0$. Second, the "error" term estimated by us is $O\left(\varepsilon^{2}\right)$, not merely $O(\varepsilon)$. Third, the conditions of our theorem do not depend on $\max \left\{\left|d f_{x}\right|: u \in \Sigma, x \in \bar{\Omega}, t \in \mathbf{R}^{+}\right\}$, while our conclusions deal with $N(t)=\max \left\{\left|d f_{x}\right|: u \in \Sigma, x \in \bar{\Omega}\right\}$. In addition, if we consider $|\Omega|$ as a parameter, then our theorem tells that the "error" term is $O\left(|\Omega|^{1 / 2+3 / m}\right)$, where $m$ is the dimension of $x$-space, since $\lambda$ is inversely proportional to the squared diameter of $\Omega[3]$.

We note as well that assumption (A-1) can be weakened to the condition that the solution of (1.1) is bounded uniformly in $\Omega \times \mathbf{R}^{+}$.

By the way, we point out that the key inequality in the proof of Theorem 2 (and hence of Theorem 3) in [5] is not true: one cannot deduce

$$
|\xi(t)| \leq \xi(0) e^{-\gamma t}=\left(N \varepsilon^{2} / \gamma\right) e^{-\gamma t}
$$

from

$$
d \xi(t) / d t \leq-\gamma \xi(t), \quad \xi(0)=-N \varepsilon^{2} / \gamma \quad((2.5) \text { of }[5]) .
$$

The correct inequality is the converse one

$$
|\xi(t)| \geq \xi(0) e^{-\gamma t}=\left(N \varepsilon^{2} / \gamma\right) e^{-\gamma t},
$$

which is useless to that proof.

4. Example. To illustrate our result, let us consider a heterogeneous reactiondiffusion system of a competitor-competitor model [4]

$$
\left\{\begin{array}{l}
\frac{\partial u_{1}}{\partial t}=\frac{1}{\varepsilon^{2}} d_{1} \frac{\partial^{2} u_{1}}{\partial x^{2}}+\left[a_{1}(x)-b_{1}(x) u_{1}-c_{1}(x) u_{2}\right] u_{1}, \\
\frac{\partial u_{2}}{\partial t}=\frac{1}{\varepsilon^{2}} d_{2} \frac{\partial^{2} u_{2}}{\partial x^{2}}+\left[a_{2}(x)-b_{2}(x) u_{2}-c_{2}(x) u_{1}\right] u_{2},
\end{array} \quad(x, t) \in(0,1) \times \mathbf{R}^{+}\right.
$$

with initial condition

$$
u_{i}(x, 0)=u_{i 0}(x), \quad i=1,2, x \in[0,1],
$$

and boundary condition

$$
\partial u_{i} /\left.\partial x\right|_{x=0,1}=0, \quad i=1,2 .
$$

Here $u_{1}(x, t)$ and $u_{2}(x, t)$ denote the population densities of two competitors with diffusion constants $d_{1} / \varepsilon^{2}$ and $d_{2} / \varepsilon^{2}$, respectively. The term

$$
\left[a_{i}(x)-b_{i}(x) u_{i}-c_{i} u_{k}\right] u_{i} \quad(i=1,2, k \neq i)
$$


represents the new growth rate of the $i$ th competitor $u_{i}$, where $a_{i}(x), b_{i}(x)$, and $c_{i}(x)$ are its intrinsic growth rate, intra- and interspecific competition coefficients, respectively. We suppose here, being the functions of spatial position $x, a_{i}(x), b_{i}(x), c_{i}(x)$ $\in \dot{c}^{1}(0,1), i=1,2$.

It is easy to check that problem (4.1)-(4.3) satisfies assumptions (A-1)-(A-3). Denote

$$
L_{i}=\max _{x \in[0,1]} \frac{a_{i}(x)}{b_{i}(x)}, \quad i=1,2,
$$

then $\Sigma=\left[0, L_{1}\right] \times\left[0, L_{2}\right]$ is an invariant region.

For simplicity, we just consider a specific case:

$$
\begin{array}{rlrl}
a_{1}(x) & =1-\left(x-\frac{1}{2}\right)^{2}, \quad a_{2}(x) & =\frac{3}{4}+\left(x-\frac{1}{2}\right)^{2}, \\
b_{1} & =b_{2}=c_{1}=c_{2}=1, \quad d_{1}=d_{2}=1 .
\end{array}
$$

This means that the only difference between $u_{1}$ and $u_{2}$ is on their intrinsic growth rate functions. They prefer growing near the center and the boundary, respectively, of the habitat.

The invariant region is $\Sigma=[0,1] \times[0,1]$ and the principal eigenvalue of $-\Delta$ is $\pi^{2}$. In addition, we have

$$
\begin{aligned}
f(x, u) & =\left[\begin{array}{c}
\left(1-\left(x-\frac{1}{2}\right)^{2}-u_{1}-u_{2}\right) u_{1} \\
\left(\frac{3}{4}+\left(x-\frac{1}{2}\right)^{2}-u_{2}-u_{1}\right) u_{2}
\end{array}\right] \\
d f_{u}= & {\left[\begin{array}{cc}
1-\left(x-\frac{1}{2}\right)^{2}-2 u_{1}-u_{2} & -u_{1} \\
-u_{2} & \frac{3}{4}+\left(x-\frac{1}{2}\right)^{2}-2 u_{2}-u_{1}
\end{array}\right], } \\
d f_{x}= & {\left[\begin{array}{c}
-2\left(x-\frac{1}{2}\right) u_{1} \\
2\left(x-\frac{1}{2}\right) u_{2}
\end{array}\right] }
\end{aligned}
$$

and hence

$$
N=\max _{\substack{x \in[0,1] \\\left(u_{1}, u_{2}\right) \in[0,1] \times[0,1]}}\left|d f_{x}\right|=\sqrt{2} .
$$

It is well known that

$$
\text { norm of } n \times n \text { matrix } A=\left(\text { greatest eigenvalue of } A^{T} A\right)^{1 / 2}
$$

and that the greatest eigenvalue of a nonnegative definite matrix is no more than the trace of the matrix. So, by a computation we get

$$
\begin{array}{cl}
M= & \max _{\substack{x \in[0,1] \\
\left(u_{1}, u_{2}\right) \in[0,1] \times[0,1]}}\left|d f_{u}\right| \\
\leq & \max _{\substack{x \in[0,1] \\
\left(u_{1}, u_{2}\right) \in[0,1] \times[0,1]}}\left\{\left[1-\left(x-\frac{1}{2}\right)^{2}-2 u_{1}-u_{2}\right]^{2}+u_{1}^{2}\right. \\
& \left.+u_{2}^{2}+\left[\frac{3}{4}+\left(x-\frac{1}{2}\right)^{2}-2 u_{2}-u_{1}\right]^{2}\right\}^{1 / 2} \\
\leq \sqrt{194} / 4 . &
\end{array}
$$


Our theorem tells that if $u_{0}(x) \in \Sigma$ and the diffusion is strong enough, namely, $\varepsilon^{2}<4 \pi^{2} / \sqrt{194}$, then the limit of $\|u(x, t)-\bar{u}(t)\|_{L^{2}}$ is no more than

$$
\sqrt{2} \varepsilon^{2}\left[\pi\left(\pi^{2}-\sqrt{194} \varepsilon^{2} / 4\right)\right]^{-1}
$$

as $t$ tends to infinity.

\section{REFERENCES}

1. K. Chueh, C. Conley, and J.Smoller, Positively invariant region for systems of nonlinear diffusion equations, Indiana Univ. Math. J. 26 (1977), 373-392.

2. E. Conway, D. Hoff, and J. Smoller, Large time behavior of solutions of systems of nonlinear reaction-diffusion equations, SIAM J. Appl. Math. 35 (1978), 1-16.

3. R. Courant and D. Hilbert, Methods of mathematical physics, Vol. 1, Interscience, New York, 1962.

4. N. Shigesada and J. Roughgarden, The role of rapid dispersal in the population dynamics of competition, Theoret. Population Biol. 21 (1982), 353-372.

5. Y. Su, Asymptotic behavior of solutions of heterogeneous nonlinear reacting and diffusing systems, Nonlinear Anal. 9 (1985), 275-288. 48109

DePARTMENT OF MATHEMATICS, UNIVERSity OF Michigan, ANN ARBOR, MichigaN

Department of applied Mathematics, Dalian institute of Technology, DAlian, PeOple's Republic of China (Current address) 